\title{
I like it but I don't have time to tell patients' families: Exploring barriers and facilitators of pain and dementia knowledge flow between healthcare providers and family caregivers
}

\author{
Cary A. Brown*, Ashley Schmidt \\ Department of Occupational Therapy, University of Alberta, Edmonton, Canada; ${ }^{*}$ Corresponding Author: cary.brown@ualberta.ca
}

Received 28 September 2012; revised 30 October 2012; accepted 10 November 2012

\begin{abstract}
Pain in people with dementia is prevalent, largely undetected, and undertreated within the care setting. Family members require resources to help them assume a more significant role in pain assessment and management. This report outlines a psycho-educational online resource developed to address these needs. The report explores the apparent disconnection between the positive evaluation healthcare providers gave the resource and their infrequent rate of referring family caregivers to the resource. We apply a sticky knowledge framework to examine these complex and incongruent findings and conclude that health literacy and knowledge translation strategies need to focus directly on family caregivers as opposed to adhering to a more traditional biomedical model in which healthcare professionals assumed the primary responsibility for gatekeeping and knowledge dissemination.
\end{abstract}

Keywords: Dementia; Pain; Knowledge

Translation; Sticky Knowledge

\section{INTRODUCTION}

If things were that simple, word would have gotten around [1].

Health literacy is a monumental challenge of the 21st century. To achieve good health people must, with more and more frequency, be able to access health self-management information, determine if it is reliable and trustworthy, understand what it means and select from the wealth of information what is relevant for themselves and their families. Family members of persons with dementia (PWD) turn to a range of sources for reliable dementia management information including healthcare professionals, service organization, friends and increasingly, the Internet. In this report dementia relates specifically to cognitive impairment, such as Alzheimer's disease, in the elderly.

Research tells us that there are many challenges to developing dementia health literacy (for example time, technology and lack of information evaluation skills) [2]. Healthcare providers are seen as reliable sources of information and, as such, are trained to assume this Knowledge Translation (KT) role during their professional education. However, healthcare providers are not uniformly able and willing to assume this role. There are numerous barriers (time, skill, awareness of need for example) and KT has become a very active research area.

This paper reports a study exploring whether targeting healthcare providers to assume a KT role in referring family members of PWD to the pain and dementia health literacy website (www.painanddementia.ualberta.ca) was a successful strategy. This information will help us design effective KT strategies for dissemination future family members of PWD health literacy resources.

\subsection{Background to the Website Evaluation}

Pain is significantly underdiagnosis and undertreatment in persons with dementia $[3,4]$. The Understanding Pain and Dementia project was created in response to family caregivers' need for pain health literacy resources related to persons with dementia and designed for both individual use and so that rural and urban community organizations could hold group workshops. The resource, grounded in evidence-based teaching strategies for working with older adults, was designed to stand alone so that a healthcare provider was not required to be present. These principles were selected to guide the project because the majority of family caregivers of PWD are older adults with age related functional consideration and unique learning needs that must be considered to maximize the effectiveness of KT initiatives [3-8]. 
The Understanding Pain and Dementia resources include an audio-visual workshop PowerPoint presentation, promotional materials, information packs, the evidencebased Pain Assessment in Advanced Dementia (PAI NAD) scale [6], a pain log, workshop evaluation forms, workshop organizer's manual, evidence-based reference materials and other pain and dementia educational resources. The resource has been made available as a free website. We made extensive efforts to raise awareness about the available pain and dementia resources among healthcare providers and families of persons with dementia. All announcements specified that the website was a pain informational resource for family members of PWD.

The electronic registration and data collection embedded in the website collects information about who used the website (family, care provider, students, etc.), the user's email address, and how they heard about the website (e.g. internet search, referral from healthcare provider, newspaper, etc.). Our preliminary utilization review showed 3203 users between 08/24/2009-12/31/2011. The majority was healthcare providers (52.8\%), followed by family members of PWD (30.8\%), and then people who work or volunteer at an organization for PWD (19.6\%). The most frequent source of referral to the website came from web searches $(28.7 \%$ in 2009 ; $37.7 \%$ in 2011) and referrals from healthcare providers (27.8\% in 2009; $17.1 \%$ in 2011). Of note was that the majority of these referrals were healthcare provider to healthcare provider, not healthcare provider to family members of PWD (Stat sig. $p<000$ ). Families were much more likely to have found the online resources using a web search. Notably, referrals by web searches increased over time while referrals by healthcare providers declined by approximately $10 \%$.

Based on these preliminary findings we carried out a more detailed follow-up of healthcare provider Registrants to determine 1) how they evaluated usefulness of the website for themselves and families of PWD, and 2) influences on their decision to refer family members to the resource.

\section{METHODS}

\section{Survey Design and Distribution}

The survey was designed to focus on understanding the relationship between healthcare providers' perceptions of the website usefulness for both their own learning needs and the learning needs of family members of PWD, and perceived barriers and facilitators to referring family members of PWD to the website. The Knowledge Translation (KT) literature consistently reinforces the need for targeted and diverse KT strategies [9-11]. To that end we include questions in the survey to gather more data about health- care providers' KT preferences. The survey was developed electronically on the Fluid-Survey ${ }^{\circledR}$ platform. FluidSurvey is a Canadian company and all data is held in Canada (http://fluidsurveys.com/canada/data-privacy-canada/). FluidSurvey allows collected data to be exported to SPSS (version 18) and Pearson's chi-squared test for independence was carried out to determine relationships between variables [12].

The 10 question survey required between 5 - 10 minutes for completion and had a grade 11.2 reading level as assessed with the Flesch-Kincaid tool imbedded in the Microsoft Word software. While a grade 11 reading level is not ideal for the general population we believe it is acceptable for healthcare providers who complete patient records and other data collection as part of their job requirements. We field tested the survey with eight health-care providers and made the minor modifications that were needed. The survey did not collect any personal identification data and we were blind to which of the healthcare providers who had registered to use the website accepted the invitation and responded to the survey invitation. Following Health Ethics Review Board approval all healthcare providers who registered to access the website received an email invitation to complete the survey. Two weeks after the original invitation, a second email was sent. Because we were blind to which registrants had responded, the email was designed to serve as both a thank you to those who had responded and a reminder for those who had not yet participated.

\section{RESULTS}

\subsection{Respondents}

Of the total possible healthcare participants (1459), 1253 email invitations to the study were successfully delivered and after initial and follow-up invitations 160 (12.8\%) responses were received. The final sample consisted of healthcare providers from Canada $(66.9 \%, \mathrm{n}=$ 107), the United States of America (15.6\%, $n=25)$, Australia $(10.0 \%, n=16)$, Europe $(3.8 \%, n=6)$, and 3.8\% (n $=6$ ) from other areas or where a location was not provided.

\subsection{Survey Questions}

As the website dissemination activities had been carried out in international media and conferences we decided to analyze the findings as a group. Some questions allowed for more than one response per category while others allowed only a single response. In addition, respondents were not required to provide responses to all questions in the survey. Consequently not all of the following findings have response rates of $100 \%$.

Q1. What is your profession? (Based on 150 completed responses): 
Nurses $-46.7 \%(\mathrm{n}=70)$, occupational therapists $-13.3 \%(n=20 / 150)$, and “other" $-10.0 \%(n=15)$.

There were few responses from pharmacists $(1.3 \%$; $n$ $=2 / 150)$ and home care workers $(0.7 \% ; n=1)$ and no responses from psychiatrists or speech language pathologists.

Q2. What is your practice setting? (Based on 152 responses):

Long-term care centers $-50.0 \%(\mathrm{n}=76)$, patients' homes $-20.4 \%(n=31)$, and in-patient acute hospitals $-19.7 \%(\mathrm{n}=30)$.

Workplaces such as geriatric day-care units $(3.3 \%, \mathrm{n}=$ $5)$ and out-patient rehabilitation facilities (2.6\%, $(n=4)$ were least frequently selected.

\section{Q3. How did you hear about the website?}

158 participants responded to this question. Fully $46.2 \%(n=73)$ stated they heard about the website using an internet search engine. The next most commonly cited sources were:

Email announcement -29.8\% ( $\mathrm{n}=47)$, and from another healthcare provider $-24.7 \%(\mathrm{n}=39)$

Q4. How frequently and for what amount of time have you used the website?

The 126 responses to this question indicate that time spent on the site was:

More than 15 minutes $-65.1 \%(n=82)$, Between 6 15 minutes $-32.5 \%(\mathrm{n}=41)$, and Less than 5 minutes $-2.3 \%(\mathrm{n}=3)$.

The section referring to the frequency of visits to the site accumulated 89 responses. Of these, 43.0\% ( $\mathrm{n}=$ 67/89) returned to the website 2 or more times.

Q5. How would you rate the usefulness of the website materials for the learning needs of healthcare providers?

Healthcare providers rated the website on a scale of no opinion (0), none is helpful (1), some is helpful (2), half is helpful (3), most is helpful (4), and all is helpful (5). Respondents rated the various materials on the website as either "most" (4) or "all” (5) helpful for their professional learning needs. Rates of response varied for each section of this question and numbers of responses within groups were as follows:

Presentation 81.2\% ( $\mathrm{n}=125)$, PainAD \& Pain Log 75.5\% ( $\mathrm{n}=114)$, Organizer's manual 51.3\% ( $=77$ ), and Participants' pack 51.0\% $(\mathrm{n}=76)$.

Q6. How do you as a healthcare provider rate the usefulness of the website materials for the learning needs of patients and their families?

Findings for the usefulness of the presentation, workshop organizer's manual, workshop participants' pack and the PainAD \& Pain Log in Question 5 were similar to responses to question 6. "Most" or "all" of the materials were rated as helpful to the learning needs of patients and their families. Rates of response varied for each section of the question and numbers of responses within groups were as follows:

Educational presentation 74.3\% $(n=113)$, PainAD \& Pain Log 62.8\% (n = 93), Organizer's manual 44.3\% (n = 66), and Participants' pack 46.9\% $(n=68)$.

A large number of healthcare providers had "no opinion" regarding the usefulness of the workshop organizer's manual (40.3\%; $n=60)$ and participants' pack $(40.0 \%$; $n=58)$ for patients and their families. It is possible that not all participants accessed these resources on the website and so, unable to comment, selected "no opinion” for their response.

Q7. Did you refer any patients/patient families to the website?

Thirty-four percent (34.1\%; $n=45 / 132)$ of healthcare provider survey respondents stated that they did refer their patients/patients families to the website and 65.9\% ( $\mathrm{n}=87$ ) stated they did not refer. Using an online Word Cloud program embedded in FluidSurvey ${ }^{\circledR}$ we were able to analyze and categorize survey responses.

Of those 45 participants who stated "yes-did refer" $73.3 \%(n=33)$ provided an explanation as to why they referred. These were grouped into four main categories (note: some reasons provided fell under more than one category making the total responses $>100 \%$ ):

1) The information is helpful/useful to patients$45.5 \%(n=15)$,

2) The information can be used to educate individuals about this aspect of dementia $-33.3 \%(n=11)$,

3) The information provides support for patients and families $-24.2 \%(\mathrm{n}=8)$, and

4) The information can be used to improve patient care $-9.1 \%(\mathrm{n}=3)$.

Sixty-five participants (74.7\%) who stated "no-did not refer” provided their reason for not referring. Six categories emerged:

1) No opportunity to make referrals $-41.5 \%(n=27)$,

2) Not applicable to current role $-21.5 \%(n=14)$,

3) Resources perceived as inaccessible to patients due to language or technology barriers $-16.9 \%(n=11)$,

4) Information used for personal learning only $-7.7 \%$ $(n=5)$ Forgot about the resources $-7.7 \%(n=5)$, and

5) Time constrains $-6.2 \%(n=4)$.

Only one response suggested that the information provided on the website was not sufficient to warrant referrals to patients or their families. Interestingly $24.6 \%$ ( $\mathrm{n}=$ $16 / 65$ ) of the respondents who had not referred patients expressed intention to do so in the future.

Q8. How can we improve the website to make it more useful for families?

Using the Word Cloud embedded in the FluidSurvey ${ }^{\circledR}$ program we categorized the suggested improvements to increase the usefulness of the Pain and Dementia Toolkit for families. 
Of the 67 responses to Question 8, 34.8\% $(n=23)$ of participants stated that they had no suggestions for improvements to the site and felt that the information provided was sufficient for families. Other responses included:

Advertise commercially or through physicians, health care providers and community centers shouldpromote resources for use by families and patients $(19.7 \%$; $n=13)$,

- Provide alternative means of retrieving the information to increase accessibility for individuals who experience language or technology barriers (10.6\%; $\mathrm{n}=$ 7). For example:

- Patients having a primary language other than English (i.e. Spanish or French)

- Poor computer literacy

- Unfamiliar with internet use

- No access to computers

- Edit the video presentation $(9.1 \% ; n=6)$ to promote smooth transitions and volume,

- Provide simplified summaries $(9.1 \% ; n=6)$, additional information $(9.1 \% ; n=6)$, provide links to other sources (4.5\%; $n=3)$, and

- Approach families to determine their specific website needs $(3.0 \% ; n=2)$.

A small number of the respondents were dissatisfied that they were required to re-register for the site with each use (4.5\%; $\mathrm{n}=3)$.

Q9. What is your preferred method of knowledge translation?

Healthcare providers were asked which method of knowledge translation would be most helpful as a reminder to provide patients families with referrals to the website resources. Respondents were asked to select their responses using a scale of least helpful (1), somewhat (2), often (3), most helpful (4), and no opinion (5). Response rates within groups varied for each segment of the question, totals for each respective question are provided within each segment:

Five out of 9 strategies rated as often helpful to most helpful were:

- Printed reminder or tip sheets to hand out during office visits $-87.0 \%(n=128)$,

- Posters/flyers for the waiting room $-2.8 \%(n=110)$,

- Email reminders from a professional organization $-70.9 \%(n=105)$,

- Regular notices in professional journals $-65.3 \%(\mathrm{n}=$ 98), and

- Endorsement from a well-known healthcare provider $-60.4 \%(n=87)$.

Three of the 9 strategies listed as somewhat helpful to often helpful were:

- Email reminders from the website designers $-57.5 \%$ (n= 84),

- Promotional items $-53.1 \%(n=77)$ for the website, and

- $\quad$ RSS feed/ online updates -42.9\% $(n=60)$

One strategy-Regular mailed reminder was most frequently $(47.5 \% ; \mathrm{n}=67)$ rated as least helpful.

Q10. How can we improve strategies for reminding healthcare providers to refer families of persons with dementia to the Pain and Dementia website?

Respondents were asked to provide, inan optional text box, their own suggestions for effective knowledge exchange strategies that would be the most benefit to help them refer families of persons with dementia to the resource.

There were 62 responses provided for this question but some responses crossed more than one category resulting in totals for this question greater than $100 \%$. The suggestions that were offered were extremely varied and the Word Cloud software was used to generate categories and organize responses.

The most frequent comment $(29.0 \%$; $n=18)$ was that agencies, such as non-profit organizations, hospitals and long term care centers, were the best source for knowledge exchange and could offer the pain and dementia information to healthcare providers through training sessions, monthly meetings and administration policy and procedure packages. Other suggestions included:

- Handing out flyers/brochures/posters $-19.4 \%(\mathrm{n}=12)$, Updating the website information $-11.3 \%$ ( $\mathrm{n}=7$ ), Em-power families to seek information from their providers $-11.3 \%(n=7)$, Improving the accessibility of the resources through language translation and more handouts $-9.7 \%(n=6)$, Establishing reminders through email or text message alerts $-8.1 \%(n=5)$, Promotional items $-6.5 \%(n=4)$, and Advertising through healthcare providers, community facilities and commercial means $-3.2 \%(n=2)$.

A few respondents noted the substantial costs required for many of these strategies. A large number of respondents made no suggestions to improvements $(21.0 \%$; $n=$ 13).

\subsection{Statistically Significant Findings}

1) Because those in nursing were the predominant participant group (46.7\%) we treated them as one group and collapsed all other participants'responses into a second group- “Other”. Chi-square analysis illustrated several statistically significant differences between the two groups. Specifically, those in nursing were more likely than other participants to:

- Be working in an institution rather than a community setting ( $p=0.021)$,

- State the pain log tool was useful for their professional learning needs ( $p=0.005)$, and

- Prefer the following strategies as reminders for providing referrals: RSS feeds ( $p=0.032)$, referrals from 
other healthcare providers $(p=0.001)$, and promotion tools $(p=0.023)$.

2) Within group chi-square analysis revealed that nonnursing ("other" category) healthcare providers were less likely than those in the nursing category to state that they heard about the website from a healthcare provider ( $p=$ 0.001 ). One possible interpretation of this is that those in nursing may have more extensive networks for information exchange in comparison to other healthcare providers.

3) All participants (including "nursing" and "others") working in institutional settings were more likely to identify that promotional items would be most helpful as a reminder of the online resources $(p=0.015)$. This group was also more likely to provide patient/family referrals to the online resources $(p=0.028)$ than those not working in this setting. One possible interpretation of this is that nursing staff in long-term/residential settings have stronger relationships with patients' families that facilitate KT activities such as this.

\section{DISCUSSION}

The findings confirmed the trend we observed at preliminary follow-up-i.e. Healthcare providers do not refer patients with dementia and their family members to the Understand Pain and Dementia website resource. They do rate the website content and resources as helpful and few participants offered critical feedback or significant suggestions for improvement. Healthcare providers are frequent users of the resource and appear to readily refer colleagues. The flow of information seems however, to bottle neck at the healthcare provider level and only $1 / 4$ stated they had referred families of persons with dementia to the website.

To use nautical imagery, the Understanding Pain and Dementia website appears to be a sound vessel when docked with healthcare providers' but anchored too strongly, unable to sail to the destination of greatest unmet need families caring for persons with dementia in the home. The following discussion will focus on exploring the elements of the website that appear to either facilitate or hinder knowledge of the website flowing to the intended audience.

Key findings from the data predominantly relate to contextual and personal characteristics that were identified by participants as barriers to the flow of information from healthcare provider to patient/family member. Most participants expressed traditional, but not necessarily evidence-based, ideas about how to best promote sharing of website information (knowledge flow) between healthcare providers and family members of PWD. For example, many participants identified preference for tip sheets and other printed handouts as helpful methods for
KT. However, research demonstrates that these methods are of questionable effectiveness, particularly when used in isolation [10].

Elwyn et al. [7] propose that information in isolation cannot affect the Knowledge Exchange (KE) process. Rather, the method of information delivery has at least an equal, if not greater, impact on the efficiency of KE.

Elwyn's model identifies specific factors that can influence resistance to knowledge flow. The idea of resistance to knowledge, concepts, and guidelines being freely exchanged between and within individuals is referred to as "stickiness" or "sticky knowledge" [7]. Stickiness is a dynamic concept which demonstrates how characteristics of information must change depending on what stage of the exchange process is active.

Stickiness in KE is analogous to glue; at the source knowledge needs to be fluid and malleable so it flows easily from source to target. At target the properties must change so that it adheres. Adherence and flow of knowledge therefore is dependent on characteristics of not only the information itself but also the source and receiving surfaces. Like glue, knowledge must flow freely from the bottle but increase in viscosity so it adheres to the target surface.

In healthcare, source and receiving surfaces are predominantly interpersonal interfaces. Flow of knowledge from source to receiver also demonstrates significant temporal and action components. Specifically, measures of the stickiness of knowledge include the ability of the target to retain, access, and act upon information appropriately as new demands arise. Elwyn et al. [7] categorize predictors of stickiness into Knowledge, Source, Recipient, and Context (see Table 1).

Knowledge: information that is exchanged to the benefit of different groups. For knowledge to be useful it must be managed competently so that the credibility of its origin and the accuracy of the information are apparent. Elywn et al. [7] suggest that within health services knowledge is unevenly distributed and unequally adopted which creates additional burdens on the healthcare system.

Source: the place or person with whom the knowledge originated. The source is generally the seed of knowledge formation and the point from which knowledge is disseminated to others. In healthcare this is often academic or clinical researchers who traditionally have been perceived as "siloed" and not well connected with knowledge users [13].

Recipient: the individual who receives and engages with the information from the source. If the recipient does not engage with the knowledge then transfer to the recipient is considered to be unsuccessful. Within healthcare recipients include a diverse range of policy makers, professional groups, academic training programs and 
Table 1. Predictors of stickiness at different points of knowledge flow process.

\begin{tabular}{clcl}
\hline $\begin{array}{c}\text { Communication } \\
\text { elements: }\end{array}$ & \multicolumn{1}{c}{ Predictors of stickiness } & Achieved & Incomplete \\
\hline Knowledge & 1.1 Causal ambiguity & Yes & \\
& 1.2 Unproven knowledge & Yes & \\
Source & 2.1 Motivation of source & Yes & \\
& 2.2 Credibility of source & Yes & \\
Recipient & 3.1 Recipient motivation & & Continuing \\
& $\begin{array}{l}\text { 3.2 Recipient absorptive } \\
\text { capacity }\end{array}$ & & Continuing \\
& $\begin{array}{l}\text { 3.3 Recipient retentive } \\
\text { capacity }\end{array}$ & & Continuing \\
4.1 Barren organizational & & Continuing \\
context & $\begin{array}{l}\text { 4.2 Arduous relationship } \\
\text { between source \& recipient }\end{array}$ & Partial & Continuing \\
\hline
\end{tabular}

Modified from Elwyn et al. [7].

members of the public. This diversity presents unique challenges for designing $\mathrm{KE}$ strategies because stakeholders will not all hold the same priorities and values [14].

Context: the circumstance and situation to which the knowledge event is related. Context of KE is dependent on accessing a gap, or what Elwyn et al call a "barren/unoccupied space”, in an organization's knowledge. This is defined as the organization's receptivity to the knowledge. For example when an organization perceives that they have no gap in knowledge there is no unoccupied space for new knowledge to flow to and adhere. Similarly, when an organization already has a plethora of policies related to a type of patient care, conceptually there is no space for new information to take hold. Within healthcare when new practices are introduced they are unlikely to flourish if not supported by both the organization and the participants who must enact the policy.

Context of knowledge transfer is also influenced by the degree to which the relationship between the source and recipient of knowledge is arduous or challenging. In healthcare the relationship between the source and the healthcare provider can be influenced by the length and closeness of relationship, ease of communication, recognition of past successes in the relationship, and the surrounding network of support that can serve to legitimize or devalue one partner in the relationship to the other. If the characteristics in the relationship are unaligned it is unlikely that KE will occur successfully [7-13]. It is noteworthy that healthcare providers often have much longer and closer relationships with their colleagues than they have with their patients. This is accentuated by the deceasing likelihood for a growing number of Canadians of having an established, long-term relationship with a primary care/family physician.

Each of these categories and characteristics of stickiness as they relate to the Pain and Dementia website will be discussed in depth in the following sections.

\subsection{Achieving Stickiness}

Encouragingly, a number of the features of the Understanding Pain and Dementia website fulfill Elwyn's criteria for predictors of knowledge stickiness (Table 1). Specifically, the review highlighted that a number of criteria related to Communication Elements categories of Knowledge and Source have been successfully met. The following section will discuss these features in detail.

\subsubsection{Knowledge}

Causal ambiguity (1.1, Table 1)

Causal ambiguity occurs when the motive between the source and the knowledge is unclear. The websites causal ambiguity is minimal as the viewer is easily able to determine that source and intended purpose align. The website information is evidence-based and the reference citations support it as trustworthy. Affiliations with the Canadian Dementia Knowledge Translation Network (CDKTN), the Alberta Centre of Aging (ACA), the Canadian Council on Learning (CCL) and the Department of Occupational Therapy at the University of Alberta are clearly displayed and website authors' pictures and voices are present in the online workshop. The purpose is unambiguous and clearly stated on the home page as being intended for family members of persons with Alzheimer's disease and other dementias. This demonstrates relevance for caregivers' own needs. Reduction in causal ambiguity is important to increase the likelihood of knowledge stickiness at the source/receiver interface.

\section{Unproven knowledge (1.2, Table 1)}

Unproven knowledge is present when a study lacks evidence to support its claims or has a reputation for being erroneous. This was not the case in the Pain and Dementia website as content is based on very clear and compelling evidence that pain in PWD is under-recognized and under-treated [15-17]. The evidence that healthcare providers receive minimal to no education about pain, especially in persons with dementia, is also strong $[3,18]$. In this regard the clear evidence-base for the website is an indicator predicting stickiness of the information.

An additional predictor of stickiness is the website's affiliation with reputable organizations, supported with citations, and links to external supplemental resources. Although many participants in the study stated they were unfamiliar with the tools provided on the website, no one questioned their credible. We interpreted this as evidence that the content was seen as reliable. 
On analysis, we now believe that targeting healthcare providers as a conduit for knowledge flow to family caregivers posed an unintended but fundamental KE barrier. Specifically, we believe that healthcare providers' lack of knowledge about the problem of pain in persons with dementia prevents them from clearly perceiving a need for information. Without a pre-existing awareness of the significant need for the information, stickiness at the healthcare provider level may have occurred but the imperative for flow from them to family members was diluted.

\subsubsection{Source}

Motivation of source and credibility of source (2.1 \& 2.2, Table 1)

The website development team was strongly motivated by clinical and research experience, as well as the compelling evidence-based that pain assessment and management is a significant unmet need leading to reduced function and increased suffering for PWD and their families. This awareness motivated us to partner for best practice in KE through links with organizations such as the Canadian Dementia Research and Knowledge Exchange (CDRAKE) organization, the Alzheimer's Society of Canada, and the ACA who have additional KE channels. This allowed the information to flow readily from the source to a number of audiences. Transparency about what the motivating factors are for a KE activity is a predictor of enhanced stickiness and the website and all dissemination activities clearly demonstrated the resource was non-profit, and health and quality of life related.

\subsection{Outstanding Barriers to Stickiness}

The Pain and Dementia website demonstrates strong predictors of stickiness in the knowledge and source elements which act to improve flow from source to recipient. However, we cannot assume this guarantees receptiveness at the source/receiver interface. Knowledge stickiness is also a function of the receiver's ability to absorb and retain the material. On analysis we found unmet predictors of stickiness for the Recipient and Context characteristics that act as barrier to the stickiness of knowledge exchange. Particularly we noted gaps (detailed below) at the level in which the information is delivered.

\subsubsection{Recipient}

Recipient motivation (3.1, Table 1)

Recipient motivation is characterized by the recipient's level of engage with the new knowledge which, in turn promotes successful knowledge flow from the source. Although the small sample size precluded drawing definitive conclusions, there was a positive recurrent theme of healthcare provider recipients being motivated to acquire pain and dementia knowledge for their own use. However, and of concern, they were less consistently motivated and able to facilitate flow of information to their patients and patients' families. Information received at the level of the healthcare provider interface became solidified, preventing further relaying of information towards patients and the patients' families.

The reasons for solidification at the level of the healthcare provider become an important barrier to knowledge exchange and closer examination to guide best practice in regards to promoting knowledge stickiness is warranted. Reasons for information solidification at the level of healthcare provider evidenced in the survey participants'comments included:

- limited opportunity to transmit the information,

- limited access to patients, and

- lack of policy implementing strategies to provide family caregiver pain and dementia resources.

Given that healthcare providers are the main avenue through which the public obtains resources about health and interventions our assumption that healthcare providers would be suitable candidates to bridge the information gap between our research and families of persons with dementia [19] appeared sound. However, it is now clear we need to give closer attention to the 2-step process we envisioned for how the pain and dementia information would flow. Stickiness predictors at both the healthcare provider receiving and relaying interfaces need to be considered. While we are confident that predictors of stickiness were in place for the flow of information between the website authors and healthcare providers, there are additional issues in the context of the interface between healthcare providers and family caregivers that need to be considered. For example, one accurate predictor of effective $\mathrm{KE}$ is the length of time two individuals have actively contributed to a relationship $[20,21]$. Quality and trust are two critical components to this relationship and can influence the effectiveness of knowledge transfer and exchange between the two parties [21]. Our finding that healthcare providers in Long-Term Care (LTC) facilities were more likely to relay website information to family members of PWD aligns with this theory. Workers in LTC facilities often have relationships with family members over extended periods of time. Whilst it is positive that pain and dementia resource knowledge flows more evidently between LTC provider and families, this was not our intended primary audience. Early, home-based intervention to reduce the risk of caregiver burden, functional deterioration and pre-mature institutionalization is a strong unmet need and we need more work to develop pain and dementia KE activities that have stickiness and flow at the level of family caregivers living in the community. 
A second characteristic that promotes stickiness of knowledge at the receiver level is health literacy. Health literacy is one's capacity to absorb, interpret and act on health information to make positive choices regarding personal health and well-being [2]. Poor health literacy is common in the Canadian population and many individuals rely on healthcare providers as their main source for information regarding health and relevant interventions $[2,22,23]$. Seniors tend to fall significantly lower in HL than younger adults. Barriers to seniors learning and $\mathrm{HL}$ are influenced by a number of factors including education, daily reading practices, internet access and training, numeracy, multiple co-morbidities interfering with new learning and cognitive tasks, and socio-economic factors such as beliefs, family back-ground and income. Many patients believe their health-care provider who will present them with all information they need. Conversely, many healthcare providers reported feeling that the quantity of patients they see during limited appointment times creates a barrier to presenting the patient with all the information needed and ensuring an accurate understanding of the message [24]. Disparity between functional levels of health literacy can also create barriers when patients and families feel overwhelmed at the quantity of information being presented by their health-care provider. In addition, health-care providers need to be more aware of the level of understanding in their patients and adjust their communication accordingly. Tools like the Newest Vital Sign (NVS) are a fast and efficient way of assessing a patient's level of health literacy prior to interactions with healthcare providers [22]. Clear knowledge of a patient's HL allows healthcare providers to tailor communication to meet the receptive capacity of the patient and family.

Healthcare providers typically act as the "gatekeeper" throughout the span of the patient/professional relationship, holding control over relevant medical information and ultimately implementing decisions for treatments and interventions [24]. There has been a strong push to provide patient-centered care such that patient and family assumes a greater role for self-management and decision-making, and the healthcare professional provides health education and capacity building to facilitate patients in this new role. However, not all healthcare providers and patients welcome and support this partnering relationship [24]. Healthcare providers with more traditional patient/provider beliefs may solidify information at the professional level and be unmotivated to enhance flow to family members. Equally, family members who prefer a more hierarchical relationship with healthcare providers will not be motivated to access and retain information for increased degrees of self-management.

When healthcare providers act as the primary decision maker but are poorly trained to recognized pain in their patients with dementia [25] this presents yet a further communication barrier and increases the risk that pain remains undiagnosed and under-treated. Ongoing study to improve recipient motivation at the level of both healthcare provider and patient/patient's family for KE should be a priority [21,22].

Recipient absorptive capacity (3.2, Table 1)

Our KE strategy to disseminate pain and dementia resources to family members of PWD through healthcare providers assumed the existence of a degree of absorptive capacity on the part of the healthcare providers. However, healthcare providers are expected to continuously absorb and retain expanding amounts of information on a variety of often diverse topics with competing priorities $[26,27]$.

These ongoing knowledge acquisition demands may result in "more breadth and less depth" and healthcare providers' need to become familiar with an ever expanding range of topics begins to compromise the depth of information they are able to retain. Healthcare providers can become saturated with new, at times conflicting, information. This saturation coupled with time constraints is a significant risk for occupational stress [28,29]. Examining the burden of information acquisition and absorption placed on healthcare professionals may provide insight as to the preferences these individuals have towards knowledge translation strategies.

Further study is needed of the cost-benefit relationship between learning about a new resource like the Understanding Pain and Dementia website as a referral source for patients' families to be more knowledgeable in health self-management as opposed to the prominent current practice of addressing pain issues as they arise. Evidence exists that eHealth patient education/self-management resources are effective and time saving for clinicians in areas such as insomnia, depression, and anxiety but, to-date, eHealth has not been well tested for family caregivers of PWD [30-32]. Consequently the value of taking additional time to familiarize one's self with a new resource can go unrecognized [31].

It is interesting that while participants recommended print materials to hand out in waiting areas would be useful, the evidence demonstrates this type of passive dissemination strategy to be of questionable value [10], Passive dissemination strategies do not address the issue of whether family members perceive resources presented on poster and in handouts to be relevant for them. Without direction from the healthcare provider family members may not recognize their own need. This is a complex situation with multiple influences on the absorptive capacity of both professionals and family members and theory development in this area is indicated.

Recipient retentive capacity (3.3, Table 1)

The retentive capacity of healthcare providers is their 
ability to retain information long-term. This can be a problem for healthcare professionals simply due to the broad range of topics they are expected to hold knowledge around. Elwyn et al. [7] propose that healthcare providers can reaches retentive capacity thus making it difficult to store new information difficult. This is termed "inert knowledge" where acquired knowledge does not come to mind when needed and thereby creates a barrier for its transmission [33]. In addition, knowledge flow is highly dependent on human judgment and it is not unreasonable to expect that information may at times be recalled or utilized incorrectly [34]. Research shows these effects to be lessened as professionals gain experience and transcribe information into long term memory storage thereby making retentive capacity and memory to action a more automatic process $[35,36]$.

\subsubsection{Context}

Barren organizational context (4.1, Table 1)

Organizational support for KE among healthcare pro-viders is required for information to have a sufficiently supportive foundation to flow through the system and achieve the degree of stickiness required to be integrated into practice. McDermott and O’Dell [37] suggest that because our culture is not built for team sharing but rather individual successes, organizations hold an influential role in enforcing knowledge sharing as part of their core values. In order to facilitate knowledge sharing some organizations employ a knowledge broker, actively selected to effectively filter and disseminate important information [37]. While some preliminary research supports the role of knowledge brokers [38] other studies have less favorable findings $[39,40]$ and more research is required.

Arduous relationship between source and recipient (4.2, Table 1)

Creating a relationship for KE between researchers and healthcare providers requires effort on both parts. Exchange of information can create a "push-pull" relationship between researchers and clinicians such that successful "push" requires researchers to recognize and highlight what features of emerging evidence are relevant to recipients. "Pull" factors from recipients include political support, policy making and general demand for the information [41]. Because both families and healthcare providers have little awareness about the prevalence of untreated pain in dementia, there is minimal "pull" created by public demand for more information. This limited amount of pull from the target audience slows the flow of information and can create a barrier to finding the information at the point it is finally desired.

The internet acts as an accessible and user friendly "information superhighway" for some people and for some it creates a significant burden as they struggle to sort information that is accurate, credible and relevant to their personal situation from the seemingly endless amount on the internet [41]. Many older adults are unfamiliar with using the internet or may have restricted access to online resources. Caregivers might not prioritize finding these resources because they do not feel that the information is needed or they underestimate the benefits these tools could offer. The Understanding Pain and Dementia website has managed to hold the number 1 (non-commercial) spot in Google search retrievals for a number of months and this helps to decrease any arduous relationship between source and recipient because novice internet users are not motivated to search beyond the 1st page of URLs retrieved [42].

The push/pull problems of healthcare KE are similar to those mentioned in marketing models which suggest that market saturation (in this case the abundance of in-formation available online), low demand (caregivers unaware that they need the information and therefore not "pulling" it from the researchers) and poor access (limited ability to access the internet) can all have an impact on the two way relationship of knowledge sharing [41]. Some respondents from our survey stated their perception that older caregivers are not computer literate. This is not necessarily the case but holding this belief, regardless of its accuracy, hindered information flow from healthcare provider to family caregivers.

Some respondents suggested organization could have their multidisciplinary teams offer this course to families as a way of improving communication and recognition of pain. This demonstrates some awareness that responsibility could be assumed at a more macro-level as opposed to assuming the responsibility for $\mathrm{KE}$ rests at the individual interface between healthcare worker and family caregiver. Lacks of standardized policies for assessment and treatment of pain that clearly involve family caregivers of PWD means that healthcare providers' practice may be restricted and not as effective. Pain assessment in dementia is dependent on observation over an extended period of time and during different activities. Without input from people who have known the person with dementia interventions at the healthcare provider level are less likely to be effective in treating the pain experienced by PWD $[43,44]$. Compounding the problem is that most healthcare providers receive minimal pain awareness training. In fact, a recent survey found that veterinarians receive 5 times more education regarding pain and treatment than physicians [25]. The same report suggests that in the upcoming 2014 Health Care Accord pain should be regarded as the fifth vital sign and should routinely be assessed and documented in patients' health records.

Implementing new policy and protocols can be made less arduous by first addressing biased beliefs and values. For example, prevalent ageist beliefs in North American 
society can motivate assessments and treatment offered to a patient based solely on age [45]. The theory of agism suggests that care rationing occurs towards older patients so that resources can be allocated to younger patients who are more likely to experience long term benefits of treatment [45]. This can affect the quality and quantity of treatment an older patient receives compared to young patients with similar diseases [45]. Similarly paternalism [46,47] can influence healthcare providers to avoid recommending certain treatments because they assume that older patients, and by extension their family caregivers, would be unwilling or unable to withstand the entire treatment process.

Finally, the relationship between source and recipient can be made more arduous when healthcare providers hold the belief that exclusive knowledge brings status and control within the patient/healthcare worker relationship. These people deliberately act to decrease the flow of information to others. This barrier to KE could be classified as knowledge hoarding or knowledge hiding depending on the individual's personal intentions $[7,48]$. There is limited information available in health literature to determine the reasoning for knowledge hoarding and knowledge hiding however, research suggests it exists and can create a significant source of added tension between the source and the recipient. It is possible that some degree of knowledge hoarding occurred with the Understanding Pain and Dementia resources as some participants in the survey reported using the resource for their personal knowledge and experience rather than sharing the resources with others.

In summary, Stickiness acts as a mode through which knowledge can be exchanged between two parties and maintained for future use. The ease through which knowledge is transmitted and recalled is not the single characteristic of stickiness that determines a successful exchange of information. Stickiness is also characterized by the Knowledge to be exchanged, the information Source, features of the Recipient, and the Context in which the information is delivered. Consideration of all factors is important for successful transmission of information between two parties. Special consideration needs to be given for tendencies of knowledge hoarding and knowledge hiding which act as a barrier to the transmission process.

\subsubsection{Assumptions of Homogeneity and Rationality}

We believe there are two additional contextual barriers not found in Elwyn et al. [7] model of predictors of knowledge stickiness and flow that need to be considered. These are what Complex Adaptive Systems (CAS) theorists call assumptions of rationality and assumptions of homogeneity. The human system is strongly influenced by multiple interacting and highly dynamic biopsycho- social factors and, consequently, emergent behaviors can be unanticipated by others and appear to be irrational [49]. Social marketing research clearly demonstrates that people are heterogeneous and decisions are strongly influenced by values, beliefs, past experience and perceptions related to present cost/benefit of any action. These features of human decision-making and behavior signpost the need for healthcare providers to abandon reductionist efforts to meet learning needs through one "best" approach.

\section{CONCLUSION}

The Understanding Pain and Dementia toolkit was designed for family caregivers in need of additional information and sources to recognize and act on the signs of possible pain in persons with dementia. From analysis of our survey findings within a sticky knowledge perspective, it appears that it may have been more efficient to target KE about the website to family caregivers directly. Further, the findings from this report suggest that there is no "one-size fits all” model of information delivery and that information should be malleable so that its stickiness and malleability adapts to the diverse needs of stakeholders. Future studies should take into consideration the methods of information delivery that target specific groups in such a way that promotes stickiness at the various stages of knowledge transfer.

\section{REFERENCES}

[1] Anon. (2012) John Derrida. Internet encyclopedia of philosophy.

http://www.iep.utm.edu/derrida/

[2] The Canadian Council on Learning (2008) Health literacy in Canada: A healthy understanding. http://www.ccl-cca.ca/ccl/Reports/HealthLiteracy.html

[3] Brown, C.A. (2010) Pain in communication impaired residents with dementia: Analysis of Resident Assessment Instrument (RAI) data. Dementia, 9, 375-389. doi:10.1177/1471301210375337

[4] Gibson, S. (2006) Older people’s pain. Pain: Clinical Updates, 14, 1-4.

[5] Alzheimer Society of Canada (2010) Rising tide: The impact of dementia on Canadian society.

http://www.alzheimer.ca/en/Get-involved/Raise-your-voic e/Rising-Tide

[6] Warden, V., Hurley, A.C. and Volicer, L. (2003) Development and psychometric evaluation of the Pain Assessment in Advanced Dementia (PAINAD) scale. Journal of the American Medical Directors Association, 4, 9-15. doi:10.1016/S1525-8610(04)70258-3

[7] Elwyn, G., Taubert, M. and Kowalczuk, J. (2007) Sticky knowledge: A possible model for investigating implementation in healthcare contexts. Implementation Science, 2, 44-52. 
[8] Hepburn, K., Lewis, M., Tornatore, J., Sherman, C.W. and Bremer, K.L. (2007) The savvy caregiver program: The demonstrated effectiveness of a transportable dementia caregiver psychoeducation program. Journal of Gerontological Nursing, 33, 30-36.

[9] Pallant, J. (2010). SPSS survival manual: A step by step guide to data analysis using the SPSS program. 4th Edition, McGraw Hill, New York.

[10] Formoso, G., Marata, A.M. and Magrini, N. (2007) Social marketing: Should it be used to promote evidence-based health information. Social Science \& Medicine, 64, 949953. doi:10.1016/j.socscimed.2006.09.022

[11] Grimshaw, J.M., Shirran, L., Thomas, R., Mowatt, G., Fraser, C., Bero, L., Grilli, R., Harvey, E., Oxman, A. and O’Brien, M.A. (2001) Changing provider behavior-An overview of systematic reviews of interventions. Medical Care, 39, Ii2-Ii45.

[12] Thompson, D.S., Estabrooks, C.A., Scott-Findlay, S., Moore, K. and Wallin, L. (2007) Interventions aimed at increasing research use in nursing: A systematic review. Implementation Science, 11, 2-15.

[13] Liebowitz, J. (2007) The hidden power of social networks and knowledge sharing in healthcare. In: Hanna, K.J. and Ball, M.J., Eds., Healthcare Knowledge Management, Springer, New York, 104-111. doi:10.1007/978-0-387-49009-0_8

[14] Nicholson, D., Artz, S., Armitage, A. and Fagan, J. (2000) Working relationships and outcomes in multidisciplinary collaborative practice settings. Child and Youth Care, 29, 39-73.

[15] Marzinski, L.R. (1991) The tragedy of dementia: Clinically assessing pain in the confused nonverbal elderly. Journal of Gerontological Nursing, 17, 25-28.

[16] Morrison, R.S. and Siu, A.L. (2000) A comparison of pain and its treatment in advanced dementia and cognitively intact patients with hip fracture. Journal of Pain and Symptom Management, 19, 240-248. doi:10.1016/S0885-3924(00)00113-5

[17] Igier, V., Mullet. E. and Sorum, P.C. (2007) How nursing personnel judge patients' pain. European Journal of Pain, 11, 542-550. doi:10.1016/j.ejpain.2006.07.003

[18] Lynch, M.E., Campbell, F.A., Clark, A.J., Dunbar, M.J., Goldstein, D., Peng, P., Stinson, J. and Tupper, H. (2007) Waiting for treatment for chronic pain-A survey of existing benchmarks: Toward establishing evidence-based benchmarks for medically acceptable waiting times. Pain Research \& Management, 12, 245-248.

[19] Haines, A., Kuruvilla, S. and Borchert, M. (2004) Bridging the implementation gap between knowledge and action for health. Bulletin of the World Health Organization, 82, 724-731.

[20] Thompson, G.N., Estabrooks, C.A. and Degner, L.F. (2006) Clarifying the concepts in knowledge transfer: A literature review. Journal of Advanced Nursing, 53, 691701. doi:10.1111/j.1365-2648.2006.03775.x

[21] Mitton, C., Adair, C.E., McKenzie, E., Patten, S.B. and Waye, P.B. (2007) Knowledge transfer and exchange: Review and synthesis of the literature. Milbank Quarterly,
85, 729-768. doi:10.1111/j.1468-0009.2007.00506.x

[22] Weiss, B.D., Mays, M.Z,, Martz, W., Castro, K.M., DeWalt, D.A., Pignone, M.P., Mockbee, J. and Hale, F.A. (2005) Quick assessment of literacy in primary care: The newest vital sign. Annals of Family Medicine, 3, 514-522. doi:10.1370/afm.405

[23] Crooks, V.A. and Agarwal, G. (2008) What are the roles involved in establishing and maintaining informational continuity of care within family practice. A systematic review. BMC Family Practice, 9, 1-9.

[24] Salmon, P. and Young, B. (2009) Dependence and caring in clinical communication: The relevance of attachment and other theories. Patient Education and Counseling, 74, 331-338. doi:10.1016/j.pec.2008.12.011

[25] Albrecht, H. (2011) Not to be Forgotten: Care of vulnerable Canadians.

http://www.pcpcc-cpspsc.com/wp-content/uploads/2011/1 1/ReportEN.pdf

[26] Mazmanian, P.E. (2006) Exploring relationships of knowledge to action. Journal of Continuing Education in the Health Professions, 26, 98. doi:10.1002/chp.56

[27] Mazmanian, P.E. and Davis, D.A. (2002) Continuing medical education and the physician as a learner-Guide to the evidence. Japan Automobile Manufacturers Association (JAMA), 288, 1057-1060. doi:10.1001/jama.288.9.1057

[28] Towe, A. (1998) Changes in healthcare and continuing medical education for the 21st century. British Medical Journal, 316, 301-304. doi:10.1136/bmj.316.7127.301

[29] Manderino, M.A., Brown, M., Peters, M. and Wirtz, R. (1994) Sources of stress for nurse practitioners. Journal of the American Academy of Nurse Practitioners, 6, 155159. doi:10.1111/j.1745-7599.1994.tb00932.x

[30] CIHI (2009) Canada's Healthcare Providers. https://secure.cihi.ca/free products/ProvProf2009EN.pdf.

[31] Espie, C.A., Kyle, S.D., Williams, C., Ong, J.C., Douglas, N.J., Hames, P. and Brown, J.S. (2012) A randomized, placebo-controlled trial of online cognitive behavioral therapy for chronic insomnia disorder delivered via an automated media-rich web application. Sleep, 35, 769-781.

[32] Spek, V., Nyklícek, I., Smits, N., Cuijpers, P., Riper, H., Keyzer, J. and Pop, V. (2007) Internet-based cognitive behavioural therapy for subthreshold depression in people over 50 years old: A randomized controlled clinical trial. Psychological Medicine, 37, 1797-1806. doi:10.1017/S0033291707000542

[33] Klein, B., Meyer, D., Austin, D.W. and Kyrios, M. (2011) Anxiety online-A virtual clinic: Preliminary outcomes following completion of five fully automated treatment programs for anxiety disorders and symptoms. Journal of Medical Internet Research, 13, e89. doi:10.2196/jmir.1918

[34] Eppler, M.J. (2007) Knowledge communication problems between experts and decision makers: An overview and classification. Electronic Journal of Knowledge Management, 5, 291-299.

[35] Bucknall, T. (2007) A gaze through the lens of decision 
theory toward knowledge translation science. Nursing Research, 56, S60-S66.

[36] Parker, J. and Coiera, E., (2000) Improving clinical communication: A view from psychology. Journal of the American Medical Informatics Association, 7, 453-461. doi:10.1136/jamia.2000.0070453

[37] McDermott, R. and O’Dell, C. (2001) Overcoming cultural barriers to sharing knowledge. Journal of Knowledge Management, 5, 76. doi:10.1108/13673270110384428

[38] Estabrooks, C. and Wallin, L. (2004) Where do we stand on the measurement of research. www.kusp.ualberta.ca/en/.../ /media/.../belfast_measurem ent.ashx

[39] Glegg, S., (2010) Knowledge brokering as an intervention in paediatric rehabilitation practice. International Journal of Therapy and Rehabilitation, 17, 203-211.

[40] Ward, V., House, A. and Hamer, S. (2009) Knowledge brokering: The missing link in the evidence to action chain. Evidence \& Policy, 5, 267-279. doi:10.1332/174426409X463811

[41] Greenhalgh, T. and Wieringa, S. (2011) Is it time to drop the "knowledge translation" metaphor. A critical literature review. Journal of the Royal Society of Medicine, 104, 501-509. doi:10.1258/jrsm.2011.110285

[42] Morahan-Martin, J.M., (2004) How internet users find, evaluate, and use online health information: A cross-cultural review. Cyberpsychology \& Behavior, 7, 497-510.

[43] Hadjistavropoulos, T., Marchildon, G.P., Fine, P.G., Herr,
K., Palley, H.A., Kaasalainen, S. and Béland, F. (2009) Transforming long-term care pain management in north America: The policy-clinical interface. Pain Medicine, 10, 506-520. doi:10.1111/j.1526-4637.2009.00566.x

[44] The Canadian Pain Society (2005) Accreditation pain standards: Making it happen. www.canadianpainsociety.ca/pdf/accreditation manual.p df

[45] Kane, R.L. and Kane, R.A. (2005) Ageism in healthcare and long-term care. Generations-Journal of the American Society on Aging, 29, 49-54.

[46] Robb, C., Chen, H. and Haley, W.E. (2002) Ageism in mental health and health care: A critical review. Journal of Clinical Geropsychology, 8, 1-12. doi:10.1023/A:1013013322947

[47] Kane, R.L. and Kane, R.A. (2001) What older people want from long-term care, and how they can get it. Health Affairs, 20, 114-127. doi:10.1377/hlthaff.20.6.114

[48] Webster, J., Brown, G., Zweig, D., Connelly, C.E., Brodt, S. and Sitkin, S. (2008) Beyond knowledge sharing: Withholding knowledge at work. Research in Personnel and Human Resources Management, 27, 1-37. doi:10.1016/S0742-7301(08)27001-5

[49] Brown, C.A. (2009) Mazes, conflict, and paradox: Tools for understanding chronic pain. Pain Practice, 9, 235-243. doi:10.1111/j.1533-2500.2009.00279.x 